\title{
Revisiter l'histoire sociale et politique de la musique années 1770 - années 1830
}

Mélanie Traversier

\section{(2) OpenEdition \\ Journals}

Édition électronique

URL : https://journals.openedition.org/ahrf/13407

DOI : 10.4000/ahrf.13407

ISSN : 1952-403X

\section{Éditeur :}

Armand Colin, Société des études robespierristes

Édition imprimée

Date de publication : 15 février 2015

Pagination : 3-11

ISBN : 978-2-200-92958-9

ISSN : 0003-4436

Référence électronique

Mélanie Traversier, «Revisiter l'histoire sociale et politique de la musique années 1770 - années

1830 », Annales historiques de la Révolution française [En ligne], 379 | janvier-mars 2015, mis en ligne le

15 février 2018, consulté le 01 juillet 2021. URL : http://journals.openedition.org/ahrf/13407 ; DOI :

https://doi.org/10.4000/ahrf.13407 


\title{
REVISITER L'HISTOIRE SOCIALE ET POLITIQUE DE LA MUSIQUE ANNÉES 1770 - ANNÉES 1830
}

\author{
Mélanie TRAVERSIER
}

En août 1776, l'Académie royale de Musique met à l'affiche Les Romans, un ballet héroïque en trois tableaux qui avait été créé quarante ans plus tôt sur un livret de Michel de Bonneval, alors Intendant des Menus Plaisirs $^{1}$. Le compositeur Giuseppe Maria Cambini est chargé d'en refaire la musique. Observateur avisé et mondain averti, le baron Grimm rend compte du spectacle avec une certaine déception :

« On a été obligé de retirer l'ouvrage après la troisième représentation. Les paroles qu'il avait prétendu faire revivre ont paru d'une insipidité parfaite ; sa composition, dont on avait pris une idée assez avantageuse sur les morceaux qu'on avait entendus de lui au Concert Spirituel et au Concert des Amateurs, n'a guère mieux réussi. On a trouvé la facture facile et passablement correcte, mais faible et froide, sans idée, sans génie $[\ldots] »^{2}$.

Guère convaincu par la partition musicale du musicien italien, Grimm en déduit le peu d'intérêt artistique et économique qu'il y a à reprogrammer une œuvre après la mort de son auteur. Au-delà de l'expression de sa désillusion, il évoque sans s'y attarder des aspects pourtant essentiels de la vie musicale parisienne dont on retrouve également les traits dans les autres capitales européennes de la musique du dernier tiers du XVIII ${ }^{\mathrm{e}}$ siècle.

(1) Sur les liens entre les Menus Plaisirs et l'Académie royale de Musique en général, et sur la carrière de Michel de Bonneval, Pauline LEMAIGRE-GAFFIER, Administrer les Menus Plaisirs. La cour, l'État et les spectacles dans la France des Lumières, Seyssel, Champ Vallon, à paraître en 2015 (livre issu d'une thèse soutenue en 2011 à l'université de Paris 1).

(2) Baron GRIMM, Correspondance littéraire, philosophique et critique, Paris, chez F. Buisson, rééd. 1812, t. 3, p. 208. 
Les institutions et lieux dédiés à la musique savante, qu'elle soit profane ou sacrée, se sont en effet multipliés et diversifiés et se singularisent peu à peu dans le paysage urbain ${ }^{3}$, notamment avec l'apparition des premières salles de concert spécifiques ${ }^{4}$; ils concurrencent les théâtres d'État plus anciens, tout en recrutant bien souvent les mêmes artistes (musiciens, chanteurs, compositeurs) qui offrent indifféremment leur talent. Les carrières se construisent ainsi selon des parcours professionnels et géographiques de plus en plus complexes qui marquent un essoufflement relatif du mécénat comme principal pourvoyeur d'emploi musical ${ }^{5}$. L'accès à une certaine réputation dépassant l'horizon de la ville de formation implique assez généralement une vie d'itinérance. Ce nomadisme est particulièrement contraignant pour les chanteurs et plus encore les chanteuses qui ne peuvent vivre de leur art que par le biais du spectacle vivant ${ }^{6}$, tandis que les compositeurs bénéficient du relais toujours plus productif et efficace de l'édition musicale gravée et des réseaux éditoriaux la soutenant à l'échelle européenne ${ }^{7}$. Les recensions du baron Grimm, dont la diffusion est certes socialement limitée, participent d'un autre processus qui valorise toujours plus le «public» comme principale instance d'accréditation culturelle, capable de lancer les carrières des plus jeunes, comme de faire tomber

(3) En particulier, sur l'insertion des lieux de musique dans le paysage urbain, Peter BORSAY, «Sounding the Town », Urban History, 29 (2002), p. 92-102 ; Juan José CARRERAS, Miguel Ángel MARÍn, Andrea Bombi (dir.), Música y Cultura Urbana en la Edad Moderna, Valencia, Instituto Valenciano de la Música, 2005 ; Hans Erich BöDEKER, Patrice VEIT et Michael WERNER (dir.), Espaces et lieux de concert en Europe, 1700-1920. Architecture, musique, société, Berlin, Berliner Wissenschafts-Verlag, 2008 ; Laure GAUTHIER et Mélanie TrAVERSIER (dir.), Mélodies urbaines. La musique dans les villes d'Europe (XVI $-X I X^{e}$ siècles), Paris, Presses de l'Université Paris-Sorbonne, 2008. Sur les implications urbanistiques, architecturales et policières liées à la multiplication des lieux de spectacle urbains aux XVIII ${ }^{\mathrm{e}}$ siècle, Christophe LOIR, Mélanie TRAVERSIER (dir.), Aller au théâtre. Pour une perspective diachronique de la circulation autour des théâtres, Antiquité -XVIII ${ }^{e}$-XIX ${ }^{e}$ siècles, Histoire Urbaine, $\mathrm{n}^{\circ}$ 38, 2013, Introduction, p. 5-18.

(4) Hans Erich BÖDEKER, Patrice VEIT et Michael WERNER (dir.), Espaces et lieux de concert, op. cit.

(5) David Hennebelle, De Lully à Mozart. Aristocratie, musique et musiciens à Paris (XVII ${ }^{e}$-XVIII ${ }^{e}$ siècles), Seyssel, Champ Vallon, 2009.

(6) Christian MEYER (dir.), Le musicien et ses voyages. Pratiques, réseaux et représentations, Berlin, BWV-Berliner Wissenschafts-Verlag, 2003. Voir également Reinhard STROHM (dir.), The Eigthteenth-Century Diaspora of Italian Music and Musicians, Turnhout, Brepols Publishers, 2001. Plus récemment encore les recherches menées depuis 2010 dans le cadre du programme ANR-DFG MUSICI dirigé par Anne-Madeleine Goulet et Gesa Zur Nieden et publiées dans Anne-Madeleine Goulet, Gesa Zur NiEDEN (dir.), Europäische Musiker in Venedig, Rom und Neapel, Actes du colloque conclusif de l'ANR-DFG MUSICI, Rome, 19-21 janvier 2012, Analecta Musicologica, $\mathrm{n}^{\circ}$ 52, 2014.

(7) En particulier, Kate VAN ORDEN (dir.), Music and the Cultures of Print, New York, Garland, 2000 ; Rudolf RASCH (dir.), Music Publishing in Europe 1600-1900, Berlin, Berliner-WissenschaftsVerlag, 2005 et Id., The circulation of Music in Europe 1600-1900, Berlin, Berliner-WissenschaftsVerlag, 2008. 
des opéras pourtant programmés sur des scènes prestigieuses ${ }^{8}$. S'il n'y pas encore de véritables périodiques savants dédiés aux seuls spectacles musicaux, la présence plus fréquente dans les gazettes et journaux d'articles relevant véritablement d'une critique musicale, et non plus seulement de la chronique des sociabilités élitaires, atteste cette mutation.

Amorcés au début du XVIII ${ }^{\mathrm{e}}$ siècle, ces phénomènes font véritablement sentir leurs effets sociaux à la fin du siècle. À ce titre, ils ont suscité un certain nombre de travaux récents, sensibles au dialogue méthodologique et heuristique entre histoire socio-culturelle et musicologie et aux échanges entre différentes historiographies nationales ${ }^{9}$. Ces recherches souvent portées par d'ambitieux programmes collectifs ont augmenté le corpus documentaire au-delà des sources musicales traditionnelles (partitions, traités sur la musique, archives des institutions musicales) et ont décrit avec finesse les modalités par lesquelles la mobilité des musiciens avait pu s'élargir et s' accélérer ${ }^{10}$; elles ont également remis en évidence la vitalité musicale des institutions religieuses et leur cohorte de musiciens et musiciennes $«$ modestes ${ }^{11}$. Analysant la hiérarchie mouvante des genres de musique savante, comme les rapports conflictuels qui peuvent opposer

(8) En particulier, parmi les travaux récents, Manuel COUVREuR, Thierry FAVIER (dir.), Le plaisir musical en France au XVII siècle, Wavre, Mardaga, 2006 ; Xavier BISARO (dir.), Les sons du théâtre - Angleterre et France (XVI ${ }^{e}$-XVIII ${ }^{e}$ siècle), Rennes, Presses universitaires de Rennes, 2013.

(9) À titre emblématique, le programme «Music Life in Europe, 1600-1900. Circulation, Institutions, Representation » soutenu par l'European Science Foundation. Sur les nouvelles orientations de la recherche consacrée à l'histoire de la musique, Jane H. FULCHER, « Defining the New Cultural History of Music, Its Origins, Methodologies, and Lines of Inquiry », dans Jane F. FULCHER (dir.), The Oxford Handbook of the New Cultural History of Music, Oxford, 2012. Ainsi, le projet ANR "Musiciens et Musiciennes des Saintes Chapelles, XIII ${ }^{\mathrm{e}}$-XVIII ${ }^{\mathrm{e}}$ siècles » (2011-2013) dirigé par David Fiala illustre la richesse de cette approche interdisciplinaire des phénomènes musicaux tels qu'ils peuvent être ressaisis dans leur épaisseur historique à partir d'une enquête collective prosopographique et d'une attention aux espaces de production et de consommation musicale.

(10) Dans le sillage des travaux menés par l'équipe de l'ANR-DFG MUSICI sur la circulation des musiciens européens vers et en Italie durant la période 1650-1750 (voir note 6), le programme européen HERA-MUSMIG dirigé par Vjera Katalinić est consacré aux cadres et effets en terme de transferts culturels de la mobilité des musiciens en Europe de l'Est, de l'Ouest et du Sud durant la période moderne : http://musmig.hypotheses.org. Sur la circulation des musiciens et des objets de culture liés au monde musical (décors, accessoires, partitions, ouvrages chorégraphiques...), ainsi que sur le rôle des réseaux qui animent le marché de plus en plus transnational de la musique en Europe, voir Mélanie TRAVERSIER (dir.), Musiques nomades : objets, réseaux, itinéraires, (Europe, XVII ${ }^{e}$ XIX ${ }^{e}$ siècles), numéro thématique, Diasporas, Circulations, migrations, histoire. $\mathrm{n}^{\circ} 26$ (à paraître, automne 2015, titre provisoire).

(11) Les travaux menés dans le cadre de l'ANR MUSEFREM, notamment à partir des archives révolutionnaires, ont récemment révélé un tissu de modestes musiciennes professionnelles, généralement organistes, qui exerçaient discrètement mais activement dans les églises et les couvents du royaume de France : Bernard DomPNIER, Sylvie Granger, Isabelle LANGLOIS, «Deux mille musiciens et musiciennes d'Église en 1790 », dans Christiane DEMEULENAERE-DOUYÈRE, Armelle LE GOFF (dir.), Histoires individuelles, histoires collectives, Sources et approches nouvelles, Paris, CTHS, 2012, p. 221-235. 
les différents métiers de la musique de manière synchronique ou diachronique, plusieurs travaux individuels ou collectifs réinterrogent notamment l'histoire musicale des Lumières et du premier $\mathrm{XIX}^{\mathrm{e}}$ siècle au prisme des gender studies ${ }^{12}$.

Les huit contributions rassemblées ici s'inscrivent dans ce renouvellement engagé depuis une dizaine d'années, tout en questionnant les habituelles frontières chronologiques académiques. Le numéro couvre en effet la période allant des années 1770 aux années 1830, dans une perspective résolument sensible à l'histoire politique heurtée de cette période et à ses effets sur le fonctionnement et les acteurs sociaux multiples du monde musical, tel qu'il s'est déjà élargi progressivement à l'échelle européenne depuis la fin du XVII ${ }^{\mathrm{e}}$ siècle. Il s'agit en effet de resituer à la croisée de l'histoire politique et de l'histoire sociale de la musique un certain nombre de dynamiques, qu'elles aient été déjà à l'œuvre ou qu'elles soient activées ou directement provoquées par les soubresauts politiques, et qui participent de la professionnalisation des métiers de la musique, de la structuration toujours plus complexe du marché de la musique et d'un mouvement croissant de politisation des œuvres musicales dont la dimension nationale ne saurait être négligée.

Révélant une autorité nouvelle en matière d'arbitrage culturel, les musiciens, à commencer par les compositeurs, revendiquent un rôle accru en terme d'expertise des talents de leurs pairs. Cette conquête

(12) Parmi les ouvrages parus dans les années 1990 et désormais classiques, on peut citer Susan MCClary, Feminine Endings : Music, Gender, \& Sexuality, Minneapolis, University of Minnesota Press, 1991, rééd. 2002 ; Marcia CITRON, Gender and the musical canon, Cambridge, Cambridge University Press, 1993 ; Ruth A. SolIE (dir.), Musicology and difference : gender and sexuality in music scholarship, Berkeley et Los Angeles, University of California Pres, 1993 ; Leslie C. DuNN,Nancy A. JONES(dir.), Embodied voices : representing female vocality in Western culture, Cambridge, Cambridge University Press, 1997. Plus récemment, sur les parcours contrastés des musiciens et musiciennes à l'époque moderne, voir l'étude consacrée aux jeunes filles formées dans les ospedali vénitiens, Caroline GIRON-PANEL, À l'origine des conservatoires : le modèle des ospedali de Venise (XVI ${ }^{\mathrm{e}} \mathrm{XVIII} \mathrm{I}^{\mathrm{e}}$ siècle), thèse d'histoire moderne, Grenoble 2-Università Ca' Foscari, 2010 (à paraître); sur les représentations sociales contraignant l'écoute de la voix féminine chantée, parmi les travaux menés par les membres du Cercle de Recherche Interdisciplinaire sur les Musiciennes (http://www.creim.fr), Raphaëlle LEGRAND, « Libertines et femmes vertueuses : l'image des chanteuses d'opéra et d'opéra-comique en France au XVIII ${ }^{\mathrm{e}}$ siècle », dans Hélène MARQUIÉ, Noël BURCH (dir.), Émancipation sexuelle ou contrainte des corps ?, Paris, L'Harmattan, 2006, p. 157-175 ; Catherine DEUTSCH, « Musique et eccellenza delle donne dans l'Italie des XVI et XVII ${ }^{\mathrm{e}}$ siècles », Actes du colloque Discours sur l'égalitélinégalité des femmes et des hommes à l'échelle européenne de 1400 à 1800 : Revisiter la Querelle des femmes, Saint-Étienne, Presses Universitaires de Saint-Étienne (à paraître) ; voir également, Sarah NANCY, $L a$ Voix féminine et le plaisir de l'écoute en France aux XVII ${ }^{\mathrm{e}}$ et XVIII siècles, Paris, Classiques Garnier, 2012. Le poids de l'imaginaire social stigmatisant la chanteuse d'opéra comme figure immorale a des implications professionnelles et judiciaires non négligeables : Mélanie TRAVERSIER, « Les chanteuses à la barre », Criminocorpus [En ligne], Musique et Justice, Les musiciens face à la justice, mis en ligne le 14 avril 2014, URL : http://criminocorpus.revues.org/2691. 
qui doit être considérée comme un marqueur de professionnalisation et d'autonomisation par rapport au système mécénal peut s'appuyer sur l'ancienne structure corporative comme à Rome (Élodie Oriol) ou au contraire s'en affranchir brutalement et précocement, comme en témoigne le déclin de la corporation des ménestriers parisiens face à l'affirmation de musiciens, souvent liés aux institutions de cour, se proclamant comme génies créateurs plutôt que comme « faiseurs de musique $»^{13}$. Les rapports entre création et professionnalisation se nouent ainsi de manière complexe, selon la plus ou moins grande institutionnalisation des activités musicales locales et selon la mainmise des autorités politiques sur la vie culturelle. La captation du discours sur la musique, sur sa nécessaire réforme ou sur les nouveaux talents musicaux par les professionnels de la musique eux-mêmes n'est toutefois pas linéaire, comme en atteste leur participation encore faible aux débats éditoriaux qui scandent à Paris la querelle des Gluckistes et des Piccinnistes et qui demeurent confisqués par les érudits et autres intellectuels écrivains ${ }^{14}$.

Ces musiciens, toujours plus professionnalisés et qui s'émancipent à terme du cadre corporatif, bénéficient d'un vivier d'emplois musicaux plus vaste en raison de la diversification et de la spécialisation des lieux de musique, qu'ils soient privés, publics ou princiers (Charlotta Wolff, David Hennebelle, Katherine Hambridge). Les horizons géographiques de leurs carrières sont également élargis, à mesure que se structure un marché européen de la musique, façonné et animé en partie par des

(13) Sur les attaques menées contre les prérogatives de la ménestrandise en France, voir Luc CHARLES-DOMINIQUE, Les ménétriers français sous l'ancien régime, Paris, Klincksieck, 1994 ; Florence GÉTREAU, « François Couperin, la Grande et ancienne Ménestrandise et Guillaume de Limoges », dans Musik. Raum. Akkord. Bild. Festschrift zum 65. Geburtstag von Dorothea Baumann. Music. Space. Chord. Image. Festschrift for Dorothea Baumann's 65th Birthday, Peter Lang, 2011, p. 163-182.

(14) Alessandro Di PROFIO, La révolution des Bouffons : l'opéra italien au Théâtre de Monsieur, 1789-1792, Paris, CNRS Éditions, 2003 ; Id., D'une scène à l'autre. L'opéra italien en Europe, Wavre, Mardaga, 2 vol., 2009. 
réseaux d'intermédiaires transnationaux ${ }^{15}$, qu'il s'agisse des éditeurs ${ }^{16}$, des diplomates ${ }^{17}$, des directeurs de salle ou des artistes eux-mêmes. Selon les ambitions et les propositions du moment, ils sollicitent tour à tour ces médiateurs de réputation et d'information musicales, ou bien activent des solidarités nationales ou professionnelles. La mobilisation de ces ressources personnelles et professionnelles n'est toutefois pas un gage de succès (David Hennebelle) et n'occulte pas les rivalités et les conflits entre gens du spectacle (Élodie Oriol, Youri Carbonnier, Catherine Authier-Menciassi).

En entraînant la disparition de certaines institutions musicales liées aux pouvoirs renversés, et par leurs effets induits en terme de recrutement des musiciens, les bouleversements politiques de la période couverte par ce numéro rendent saillantes ces tensions et rappellent la précarité du statut de musicien. Offrant d'abord des opportunités inespérées à de nouveaux venus, les théâtres d'État et les autres lieux princiers de représentations musicales, au-delà de leur changement temporaire de dénomination officielle, recomposent assez vite leurs rangs, non sans

(15) Sur l'histoire récemment renouvelée des réseaux, attentive aux acteurs, aux usages, aux champs d'intervention des réseaux, citons en France les travaux lancés dans le cadre de l'ANR CITERE animée par Pierre-Yves Beaurepaire, qui trouvent des échos parmi les jeunes chercheurs (groupe RES-HIST ; laboratoire Junior NHumérisme ; atelier REPLIC à l'université Bordeaux 3), les recherches animées par Carole Carribon et Dominique Picco articulant histoire des réseaux et histoire des femmes : Fanny BUGNON, Carole CARIBBOn, Bernard LACHAISE, Delphine DUSSERT-GALINAT, Dominique PICCO (dir.), Femmes et réseaux dans les sociétés modernes et contemporaines. Réalités et représentations, Actes du colloque de Bordeaux, 16-17 octobre 2014, Presses universitaires de Bordeaux, (à paraître fin 2015) ; voir également sur le cas particulier des réseaux animant le marché musical européen sur la longue durée, Mélanie TRAVERSIER (dir.), Musiques nomades, op. cit ; à l'étranger, les récents ateliers de travail du programme Analisi delle Reti Sociali (notamment celui des 20-22 juin 2013, consacré aux « Networks in space and time : Models, Data collection and Applications », et ceux de 1'Historical Network Research (Gand,15-19 Septembre 2014).

(16) Voir par exemple sur le rôle des éditeurs allemands installés à Paris et liés au milieu musical, Joann ELART, « Une nouvelle voie pour la circulation musicale : l'exemple des symphonies de Sterkel entre France et Allemagne (1777-1783 »), Dix-Huitième siècle, n 43, 2011, p. 101-130.

(17) Sur le rôle des diplomates comme médiateurs culturels dans l'Europe des Lumières, en particulier dans le cadre des transferts culturels liés aux arts de la scène, Charlotta WoLFF, «L'ambassadeur des spectacles : le comte de Creutz et l'opéra », dans Pierre-Yves BEAUREPAIRE, Rahul MARKovits, Mélanie TraVERsier, Charlotta WOLFF (dir.), Les circulations musicales et théâtrales, vers 1750-vers 1815. Actes du colloque international de Nice, 21-23 novembre 2014, à paraître ; Rahul MARKoviTs, Civiliser l'Europe. Politiques du théâtre français au XVIII siècle, Paris, Fayard, 2014 ; Mélanie TRAVERSIER, «Costruire la fama : la diplomazia al servizio della musica durante il Regno di Carlo di Borbone », dans Anne-Madeleine GouleT, Gesa ZuR NIEDEN (dir.), Europäische Musiker, op. cit., p. 171-189. Voir également, sur les expressions diverses du rôle culturel des diplomates, les contributions réunies dans Rebekah AHRENDT, Mark FERRAGUTO, Damien MAHIET (dir.), Music and Diplomacy from the Early Modern Era to the Present, Palgrave Macmillan, décembre 2014. Pour la période contemporaine, voir le numéro dirigé par Isabelle DASQUE et Renaud MELTZ, Histoire, Économie et Société, $\mathrm{n}^{\circ}$ 2014/2, Pour une histoire culturelle de la diplomatie. Pratiques et normes diplomatiques au XIX $X^{e}$ siècle, ainsi que le double numéro thématique dirigé par Anaïs FLÉCHET et Antoine MARÈS, Relations internationales, $\mathrm{n}^{\circ} 155-156$, Musique et relations internationales I et II, $2013 / 3$ et $2014 / 1$. 
conflits entre vrais anciens, vrais parvenus, faux et fausses nouvelles recrues (Philippe Bourdin, Youri Carbonnier). Si du point de vue du calendrier des saisons musicales, des effets de prestige recherchés et des musiciens engagés, un certain nombre de permanences sont manifestes entre les institutions musicales d'Ancien Régime et celles relevant d'un nouvel ordre politique révolutionnaire ou impérial (Katherine Hambridge), le renforcement de la vocation explicitement politique de la musique en tant qu'elle vise à conquérir les coeurs et convaincre les esprits se lit dans certaines formes musicales inédites ou renouvelées. C'est le cas notoire des chansons et hymnes révolutionnaires, mais aussi des œuvres savantes résolument patriotiques, créées au service de la cause révolutionnaire ou de la gloire du nouveau pouvoir, des figures ou des événements emblématiques qui l'incarnent (Joann Élart). Ce processus de politisation de la musique ${ }^{18}$ ne submerge pas l'ensemble des évolutions amorcées ou entérinées au $\mathrm{XVIII}{ }^{\mathrm{e}}$ siècle, il peut les accélérer ou les freiner non sans a-coups, selon des rythmes variés qui sont en partie calqués sur les spécificités politiques et culturelles locales héritées et présentes (Katherine Hambridge). Les années suivant le Congrès de Vienne sont marquées par le renforcement du caractère concurrentiel et internationalisé du marché de la musique profane, la concurrence accrue entre lieux et institutions programmant des manifestations musicales, la complexification des carrières et des itinéraires professionnels transnationaux et définitivement par la promotion de la voix de femme sur les scènes européennes et bientôt mondiales. Les plus célèbres chanteuses d'opéra sont enfin en partie libérées du soupçon moral ayant longtemps entaché leur destin musical et peuvent s'attirer l'éloge le plus exalté. Ainsi, le 9 septembre 1816, le marquis de Custine, saisi d'admiration « jusqu' à la terreur » après avoir entendu « La » Catalani à Francfort, écrit aussitôt à son amie Rahel Varnhagen, la brillante femme de lettres dont le salon berlinois est fréquenté par la fine fleur des artistes et auteurs allemands :

(18) Sur les appropriations politiques des formes musicales dans les années révolutionnaires et plus généralement les rapports entre musique et politique depuis la fin du XVIII ${ }^{\mathrm{e}}$ siècle, voir Esteban BuCH, La Neuvième de Beethoven - Une histoire politique, Paris, Gallimard, 1999 ; Martin KALTENECKER, La Rumeur des batailles, Paris, Fayard, 2000 ; Mélanie TRAVERSIER, « Transformer la plèbe en peuple ». Théâtre et musique à Naples en 1799, de la proclamation de la République napolitaine à la Première Restauration ", $A H R F, \mathrm{n}^{\circ} 335,2004$, p. 37-70, et plus récemment, pour un observatoire contemporain, Esteban BUCH, « L'Orchestre de Paris et Daniel Barenboïm dans l'Argentine du général Videla : la musique et le silence de la mort », Relations internationales, $\mathrm{n}^{\circ} 156$, Musique et relations internationales II, op. cit., p. 87-108. 
«Mme Catalani chante, ou plutôt laisse chanter par sa bouche le démon de l'art [...]. Elle vous délie ou vous enchaîne à son gré en se jouant toutà-tour de l'impression qu'elle produit et de celle qu'elle éprouve ; vous n'êtes plus en vous, vous êtes dans la musique, la musique est en vous, c'est un talent immédiat qui vous plonge dans la source ; impossible d'y penser, impossible de la juger, c'est le torrent de ce chant qui se précipite d'un autre monde dans celui-ci et qui entraîne tout avec lui [...]. Ah ! venez, venez l'entendre, cherchez-la, suivez-la, poursuivez-la $[\ldots] »^{19}$.

Rahel Vanhagern ne manque pas de suivre la suggestion de son correspondant. Si elle s'exprime laconiquement, son « enchantement » est tout aussi évident : «J'ai entendu la Catalani ; ici je n'ajoute rien, je répète tout ce que vous avez dit $»^{20}$.

Quelques années plus tard, les succès parisiens et les anecdotes plus ou moins légendaires et bienveillantes qui contribuent à la célébrité ${ }^{21}$ de la chanteuse italienne Giuditta Pasta (Catherine Authier-Menciassi) marquent à coup sûr l'avènement des femmes soprani au sommet de la hiérarchie vocale ${ }^{22}$; plus largement, les collaborations parfois houleuses mais fructueuses entre Gioacchino Rossini ${ }^{23}$ et Giuditta Pasta illustrent de manière exemplaire ces réalités « nouvelles » et durables du marché de la musique, les contraintes comme les opportunités financières, publicitaires et statutaires qui en résultent pour les artistes d'opéra en particulier. D'autres processus socio-économiques viennent par la suite accentuer les caractères propres à ces carrières musicales professionnelles : la «mondialisation » des carrières avec l'émergence d'un marché « américain » de la musique lié aux acteurs sociaux de la vie musicale européenne, l'affirmation et le respect du droit d'auteur des compositeurs qui vont de pair avec l'explosion et la spécialisation critique de l'édition musicale, l'institutionnalisation de

(19) Lettres du Marquis A. De Custine à Varnhagen d'Ense et Rahel Varnhagen d'Ense, Bruxelles, C. Muquardt, 1870, lettre du marquis A. de Custine à Rahel Varnhagen, datée du 9 septembre 1816, p. 55-61.

(20) Ibid., lettre de Rahel Varnhagen au marquis A. de Custine, datée du 9 décembre 1816 (Karlsruhe), p. 78-83:83.

(21) Sur la construction et les usages sociaux de la célébrité, voir Fred INGLIS, A Short History of Celebrity, Princeton, 2010 et le numéro thématique du Lapham's Quarterly, Celebrity (winter 2011) ; et plus récemment encore, Antoine LILTI, Figures publiques. Célébrité et modernité (1750-1850), Paris, Fayard, 2014.

(22) Rachel CoGWILl, Hilary PORRIS (dir.), The Arts of the Prima donna in the Long Nineteenth Century, Oxford, Oxford University Press, 2012.

(23) Sur la carrière européenne de Rossini et ses étapes parisiennes en particulier, Aurélie BARBUSCIA, Rossini et la «Restauration » de la grandeur musicale dans la France des années 1820, thèse d'histoire et civilisation, soutenue à l'Institut Européen de Florence, 2013. 
l'histoire de la musique ${ }^{24}$, l'essoufflement relatif de la musique sacrée et la promotion générale et effective des musiques nationales ${ }^{25}$ en même temps que l'affirmation d'un « grand répertoire » européen...

Ce numéro qui ne prétend nullement dresser un panorama exhaustif de la vie musicale européenne au tournant des XVIII ${ }^{\mathrm{e}}$ et $\mathrm{XIX}^{\mathrm{e}}$ siècles, explore donc quelques-uns et quelques-uns seulement des terrains de la recherche la plus récente telle qu'elle articule toujours plus fructueusement histoire et musicologie. En se focalisant sur une période marquée par la transition entre ce que la taxinomie musicologique appelle le classique et le romantisme, les contributions rassemblées rendent d'autant plus visibles les questions relatives à la dimension sociale et politique de l'histoire de la musique.

Mélanie TRAVERSIER

IRHiS-UMR 8529

Université de Lille 3

Rue du Barreau

BP 60149

59653 Villeneuve d'Ascq Cedex melanie.traversier@wanadoo.fr

(24) On songe notamment à l'entreprise « encyclopédique » de François-Joseph Fétis : sur son œuvre de musicographe et la genèse de la musicologie savante, Rémy CAMPOS, François-Joseph Fétis, musicographe (1784-1871), Genève, Droz, Haute École de Musique de Genève, 2013.

(25) Voir en particulier Didier FRANCFORT, Le Chant des Nations. Musiques et Cultures en Europe (1870-1914), Paris, Hachette Littératures, 2004 ; Philipp THER, In der Mitte der Gesellschaft. Operntheater in Zentraleuropa 1815-1914, Vienne-Munich, Oldenbourg, 2006. 